\title{
All-optical Phase Regeneration with Record PSA Extinction Ratio in a Low-birefringence Silicon Germanium Waveguide
}

\author{
Mohamed A. Ettabib, Member, OSA, Kyle Bottrill, Member, OSA, Francesca Parmigiani, Member, IEEE, \\ Alexandros Kapsalis, Adonis Bogris, Member, OSA, Mickael Brun, Member, IEEE, \\ Pierre Labeye, Member, IEEE, Sergio Nicoletti, Member, IEEE, Kamal Hammani, Member, IEEE, \\ Dimitris Syvridis, David J. Richardson, Fellow, IEEE, Fellow, OSA, and \\ Periklis Petropoulos, Member, IEEE, Senior Member, OSA
}

\begin{abstract}
We report a low-power continuous wave (CW)pumped phase sensitive amplifier (PSA)-based phase regenerator implemented in a passive silicon-based waveguide. A polarization assisted (PA)-PSA, consisting of two orthogonally-polarized pumps and a phase-locked signal co-polarized to one of them, was implemented in a low-birefringence silicon germanium ( $\mathrm{SiGe})$ waveguide. The strong TE/TM modal symmetry of the waveguide and its large nonlinear coefficient enabled the achievement of an extremely large phase sensitive extinction ratio (PSER) of approximately $29 \mathrm{~dB}$ for a total input power of only $21.3 \mathrm{dBm}$. This SiGe-based PSA was used to demonstrate phase regeneration on a $20 \mathrm{~Gb} / \mathrm{s}$ differential phase-shift keying (DPSK) signal, thereby reducing its error vector magnitude (EVM) and phase error by three and six times respectively and enabling a bit-error ratio (BER) improvement of up to $2 \mathrm{~dB}$.
\end{abstract}

Index Terms-Silicon Photonics, Four-Wave Mixing, Phase Shift Keying, Optical Waveguide, Integrated Optics, Nonlinear Optics.

\section{INTRODUCTION}

$\mathbf{I}$ $\mathrm{N}$ recent years, optical communication networks have had to constantly adapt to a sustained growth in network traffic, driven by the rise of high bandwidth applications and the increased number of network users [1]. For instance, the once standard and simple on-off keying (OOK) data signaling is now being replaced by increasingly more complex modulation formats, such as differential phase-shift keying (DPSK),

This work was supported by the European Communities Seventh Frame-work Programme FP7/2007-2013 under Grant 288304 (STREP CLARITY) and the Photonics Hyperhighway Programme Grant (EPSRC grant EP/I01196X). The data for this paper can be found at DOI: $10.5258 / \mathrm{SOTON} / 397445$.

M. A. Ettabib, K. Bottrill, F. Parmigiani, D. J. Richardson and P. Petropoulos are with the Optoelectronic Research Centre, University of Southampton, SO17 1BJ, Southampton, United Kingdom (email: mae206@orc.soton.ac.uk, krhb1g12@soton.ac.uk, frp@orc.soton.ac.uk, djr@orc.soton.ac.uk,pp@orc.soton.ac.uk).

A. Kapsalis, A. Bogris and D. Syvridis are with Department of Informatics and Telecommunications, National and Kapodistrian University of Athens, Panepistimiopolis, Ilissia, 15784, Athens, Greece (email: alex@di.uoa.gr, abogris@di.uoa.gr, dsyvridi@di.uoa.gr). Adonis Bogris is also with the Department of Informatics, Technological Educational Institute of Athens, Aghiou Spiridonos, Egaleo, 12243, Greece.

M. Brun, P. Labeye, S. Nicoletti are with CEA-Leti MINATEC Campus, 17 rue des Martyrs 38054 Grenoble Cedex 9, France (email: mickael.brun @ cea.fr, pierre.labeye@cea.fr, sergio.nicoletti@cea.fr)

$\mathrm{K}$. Hammani is with Laboratoire Interdisciplinaire Carnot de Bourgogne, UMR 6303 CNRS-Universit de Bourgogne Franche-Comt, 9 av. A. Savary, BP 47870, 21078 Dijon Cedex, France (email: kamal.hammani@u-bourgogne.fr) quadrature phase shift keying (QPSK) and quadrature amplitude modulation (QAM), in order to increase the network's spectral efficiency and the overall system capacity.

However, both linear and nonlinear phase noise represent a major obstacle in the processing of those phase-encoded signals and can lead to significant limitations in system performance. Phase sensitive amplifiers (PSAs) have been suggested as a promising platform for phase-processing applications due to their potential for achieving noiseless amplification [2], phase regeneration [3] and quantization [4]. Almost-ideal binary step-like phase functions may be achieved for large nonlinear phase shifts, where the in-phase components of an incoming electric field are amplified, while its quadrature components are attenuated, thus facilitating phase regeneration of phase-encoded signals [5].

PSAs are commonly implemented using highly nonlinear fibers (HNLFs) [6] that are typically hundreds of meters long and enable the achievement of large nonlinear phase shifts. However, the performance of HNLFs is often limited in practice both by the onset of stimulated Brillouin scattering (SBS) which restricts the amount of input power that can be launched into them, as well as by variations in the zero dispersion wavelength (ZDW) along the fiber length which limits their bandwidth.

An alternative $\chi^{(3)}$-based platform to HNLFs offering substantial benefits in terms of compactness and the potential for further integration is silicon-based waveguides and nanowires. Silicon photonics technology has attracted significant academic and industrial research attention in recent years resulting in rapid growth and increasing maturity [7]. The high linear and nonlinear refractive indices of silicon allow a tight mode confinement and strong nonlinear interaction, making silicon devices highly attractive for nonlinear applications [8]-[10]. The broadband transparency of silicon also makes it a suitable candidate for a wide range of applications and wavelengths [11], [12]. Furthermore, the ability of silicon to be mixed with other III-V elements such as Germanium (Ge) can impact a host of its optical properties such as loss, nonlinearity, twophoton absorption (TPA) and dispersion [13]. This allows a route towards engineering devices depending on the targeted application and resulting in a highly versatile nonlinear platform [14]-[16]. 
However, despite the numerous and impressive demonstrations of various all-optical signal processing applications at telecommunication wavelengths, PSA demonstrations in silicon-based nonlinear devices have been relatively limited. Recent reports have demonstrated the achievement of a phase sensitive extinction ratio (PSER) of $11 \mathrm{~dB}$ based on pumpdegenerate FWM in a dispersion-engineered silicon photonic crystal $(\mathrm{PhC})$ waveguide by using pulsed pumps and taking advantage of the slow-light-enhanced nonlinearity [17]. Furthermore, a p-i-n junction across a silicon waveguide was used in [18] to decrease the nonlinear losses introduced by freecarrier absorption (FCA), allowing phase-sensitive extinction ratios of $20 \mathrm{~dB}$ to be reached under continuous-wave $(\mathrm{CW})$ pumping operation. Both of these PSA demonstrations have relied on scalar PSA schemes (where the signal and the pumps are co-polarized) using waveguides which exhibited TE-mode preference. As such, this necessitated either the use of impractically high amounts of pump power or active nonlinear loss suppression to realize large nonlinear phase shifts and thus achieve high PSERs.

We have recently reported the first low birefringence siliconbased waveguide $\left(\mathrm{Si}_{0.8} \mathrm{Ge}_{0.2}\right)$ exhibiting strong TE/TM mode similarity across a broad wavelength range as well as a large nonlinear coefficient [19]. The low birefringence of the waveguide enable the use of a vector PSA configuration (crosspolarized pumps), in which a polarization-assisted (PA)-PSA scheme (where the signal is co-polarized with one of the pumps) [20], [21] can be implemented. To this end, in this paper we expand on the work reported in [22] and [23], and demonstrate the first passive and CW-pumped PSA in a silicon-based waveguide exhibiting a record PSER of 28.6 $\mathrm{dB}$ for a total input power of $21.3 \mathrm{dBm}$. We exploit this large PSER to successfully regenerate the phase of a 20$\mathrm{Gb} / \mathrm{s}$ DPSK signal. A six-fold reduction in phase-noise and a bit-error ratio (BER) improvement of approximately $2 \mathrm{~dB}$ in the regenerated signal for the most severe case of noise levels examined is observed. We present a discussion on the birefringence properties of the waveguide and how these were achieved, and provide details on the PSA implementation as well as the methodology followed in our measurements.

\section{Polarization-Assisted Phase Sensitive AMPLIFICATION}

Figure 1 shows the operating principle of the PA-PSA scheme. It is based on a signal-degenerate parametric process characterized by two orthogonally polarized pumps. The signal is phase-locked to the pumps and co-polarized with one of them, which results in an idler generated in the orthogonal polarization. It follows then, that if the signal and idler are analyzed separately, no interference between them will be observed, i.e. no phase-sensitive amplification will take place. However, by rotating the transmission axis of a polarizer placed at the output of the parametric amplifier by a certain angle with respect to the idler's polarization axis (the exact value of which is dictated by the strength of the generated idler and thus, the pump power), it is possible to exactly match the powers of the signal and idler projections on this axis. This

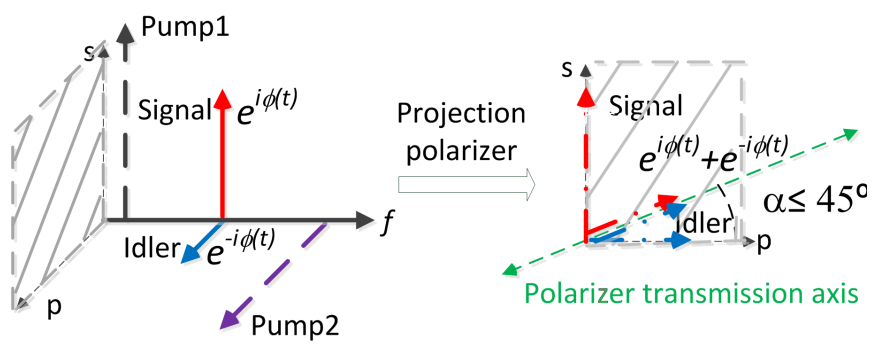

Fig. 1. Operation principle of the polarization assisted PSA scheme [21]. Left: state of polarization of the various waves in the PSA. Right: angular projection of the polarizer at the output.

is the condition for operating the PSA at maximum gain and leads to the observation of a large PSER [20], [21]. A key requirement of a polarization-assisted PSA is the presence of a low-birefringence nonlinear medium, so that efficient parametric mixing can be achieved between synchronously propagating orthogonal waves.

\section{Low BiREFRINGEnce SiGe WAVEguides}

With the requirement for a low-birefringence nonlinear medium in mind, a waveguide was designed targeting a high nonlinearity and low loss at the same time. A full waveguide design analysis was carried out by means of a Finite Element Method (FEM) solver and considered two waveguide structures: an embedded waveguide in a silicon encapsulation and an air-clad waveguide structure [19].

It was found that a strip waveguide structure embedded in a silicon encapsulation would yield these design targets and thus be suitable for implementing the PA-PSA scheme. Practical considerations relating to the fabrication process forced us to fix the waveguide height to $1.7 \mu \mathrm{m}$. Then the impact of the waveguide width on nonlinearity, dispersion and birefringence was analyzed. The analysis found that a range of waveguide widths between 0.7- $2.2 \mu \mathrm{m}$ displayed very similar transversal modal field components $\left(\mathrm{E}_{x}\right.$ and $\left.\mathrm{E}_{y}\right)$ and thus the low birefringence properties targeted [19]. Among the

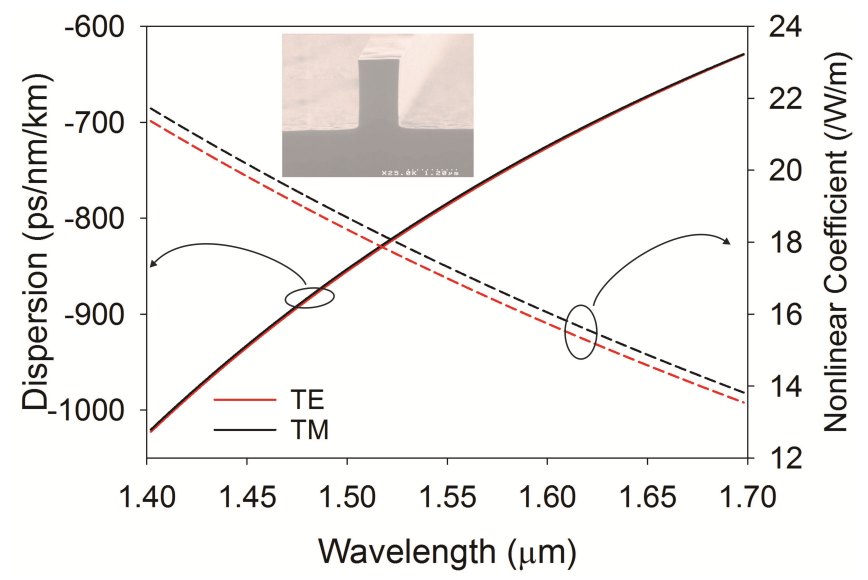

Fig. 2. Dispersion and nonlinear coefficient curves for the structure under investigation as a function of wavelength. Inset: SEM image of the $1 \mu \mathrm{m}$ wide $\mathrm{SiGe}$ strip waveguide before encapsulation in a $12 \mu \mathrm{m}$ Si cladding layer. 


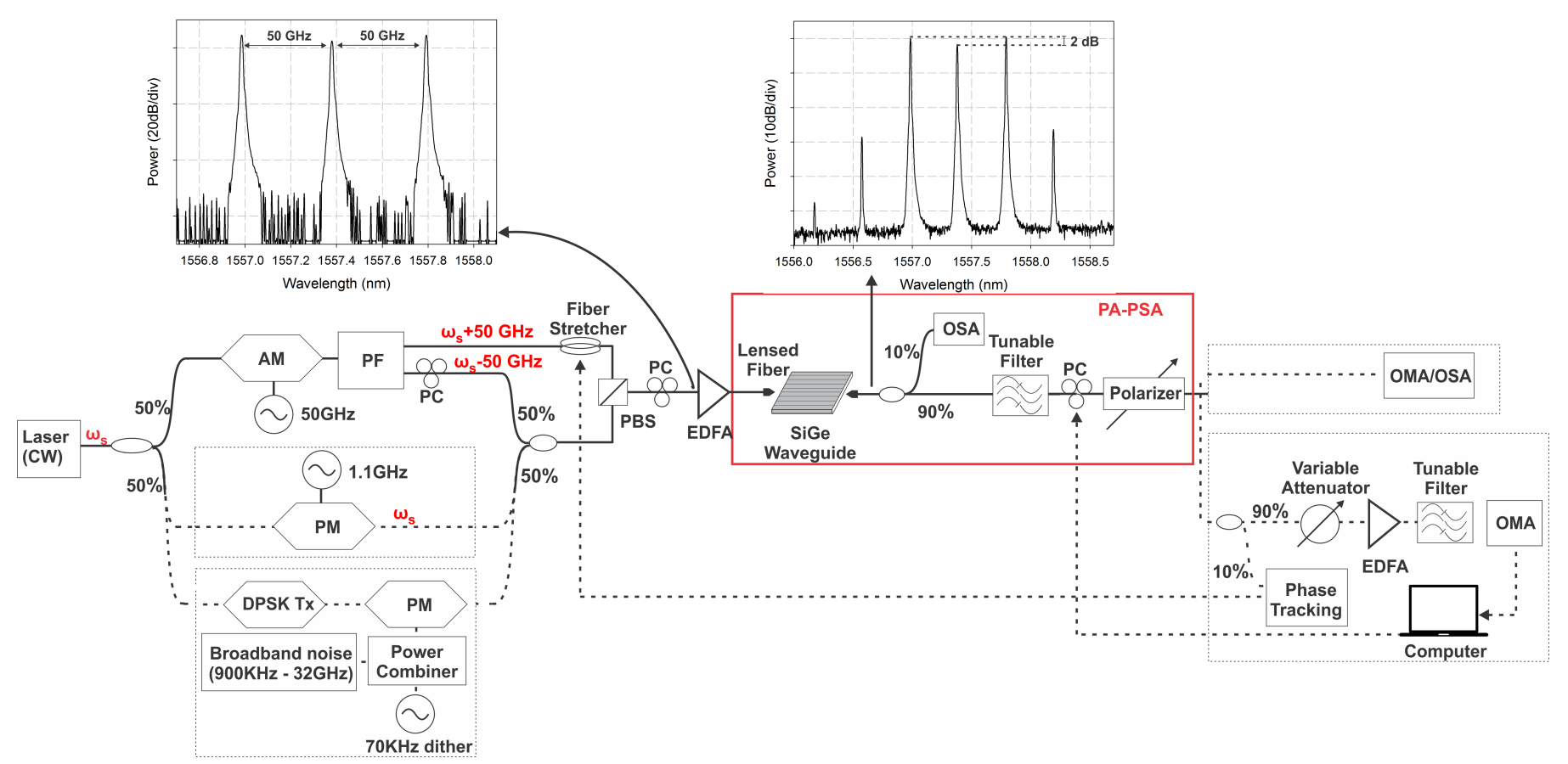

Fig. 3. Experimental set-up. Inset figures: spectral traces at the input and output of the PSA.

viable design choices, the $1 \mu \mathrm{m}$ width waveguide was found to exhibit the best combination of nonlinearity, linear loss and coupling loss and as such, it was eventually chosen for the implementation of the polarization-assisted PSA.

The full fabrication process of the waveguide has previously been reported in detail in [13]. The inset of Fig. 2 displays a scanning electron microscope (SEM) image of the waveguide cross-section before encapsulation. The length of the waveguide was $20 \mathrm{~mm}$. Coupling was facilitated through a $2-\mu \mathrm{m}$ taper at its entrance. The propagation loss of the fabricated waveguide was estimated to be $1.4 \mathrm{~dB} / \mathrm{cm}$ for both TE and TM modes, while the nonlinear coefficient was estimated to be 17 $/ \mathrm{W} / \mathrm{m}$ and $17.3 / \mathrm{W} / \mathrm{m}$ for the TE and TM modes respectively. The calculated dispersion curves as well as the nonlinear coefficient of the two polarization axes of the waveguide over a wide wavelength region around $1.55 \mu \mathrm{m}$ are shown in Fig. 2 . As can be seen in the figure, the two polarization modes were predicted to exhibit very similar dispersion and nonlinearity across the $1.40-1.70 \mu \mathrm{m}$ band.

\section{EXPERIMENTAL SETUP AND RESUlTS}

\section{A. Phase Sensitive Extinction Ratio Measurement}

Figure 3 shows a schematic of the experimental set-up that was implemented for the characterization of a PA-PSA based on the low-birefringence SiGe waveguide. An amplitude modulator (AM) was used to modulate a component of a 1557.4-nm CW beam and generate an optical frequency comb with $50 \mathrm{GHz}$ separation. The optical comb was subsequently coupled to a programmable filter (PF) (Finisar Waveshaper $4000 \mathrm{E}$ ) to select and equalize two frequency lines with 100 $\mathrm{GHz}$ separation which acted as the two pump waves. The other component of the $\mathrm{CW}$ laser beam was passed through a phase modulator (PM) driven by a sinusoidal signal at $\sim 1.1 \mathrm{GHz}$ (note that this choice of frequency is not significant and related to the operation characteristics of our measuring instruments). This produced an optical signal that was phase-locked to the pumps and whose optical phase traversed the full $2 \pi$ range. Experimentation with this signal allowed us to qualitatively assess the phase squeezing characteristics of the PSA through observation of a polar phase diagram on an optical modulation analyzer (OMA). The three optical lines were then recombined by means of a polarization beam splitter (PBS), ensuring that the two pump waves were orthogonal to one another. In this characterization the PSA was operated in free-running mode without a feedback phase-locking circuit. The three waves were amplified using an Erbium-doped fiber amplifier (EDFA) and launched into the $\mathrm{Si}_{0.8} \mathrm{Ge}_{0.2}$ waveguide using a lensed fiber with a $2 \mu \mathrm{m}$ spot size, resulting in approximately $8 \mathrm{~dB}$ coupling loss and a total coupled power of $21.3 \mathrm{dBm}$. This power level is well below the two-photon absorption (TPA ) and free-carrier absorption thresholds estimated for a similar waveguide [13]. Typical conversion efficiencies (CEs) at this power level were $\sim-22 \mathrm{~dB}$. The signal and the orthogonally generated idler at the same frequency were subsequently filtered and their polarization state adjusted using a polarization controller (PC) to exactly match the strength of their projections on the axis of the subsequent polarizer, resulting in a large PSER.

The optical-signal-to-noise ratio (OSNR) of the output signal for maximum gain was more than $30 \mathrm{~dB}$. The PSER of the PSA was measured by observing the maximum and minimum phase-sensitive gain on an optical spectrum analyser (OSA). This is normally achieved by precisely controlling the relative optical phase difference between the pump and the signal waves, so as to ensure operation in either of these conditions. In our case however, this complexity was avoided 
by allowing the PSA to run freely, thereby traversing between the two extreme gain values over time. By setting the OSA on either the maximum-hold or the minimum-hold values and allowing it to sweep the spectrum multiple times, we were able to record the conditions for maximum and minimum phasesensitive gain respectively (Fig.4). Using this method, a PSER value of $28.6 \mathrm{~dB}$ was measured. (It is noted that obviously this discussion does not apply for the noise floor, which is indeed shown to be different in the two holding modes in Fig. 4).

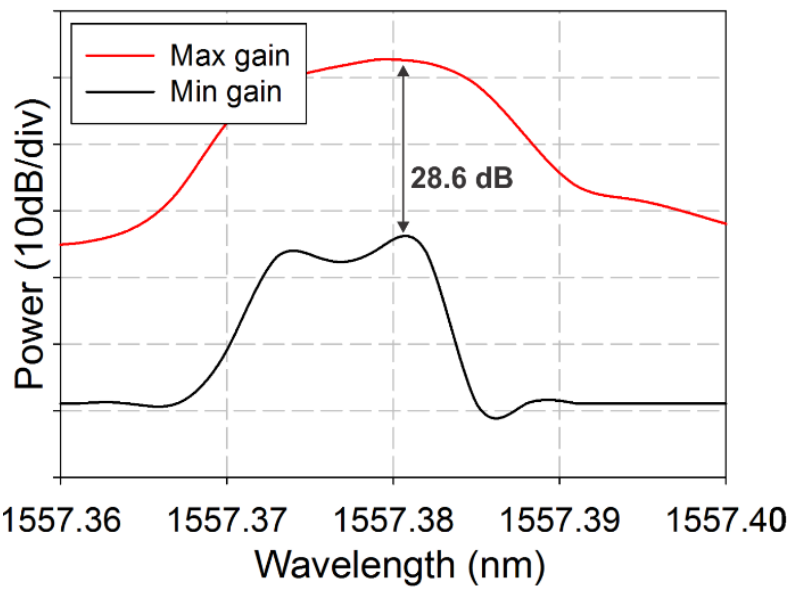

Fig. 4. Optical spectra corresponding to the maximum and minimum gain.

To assess the benefit obtained by adopting the PA-PSA configuration, the waveguide was also assessed in a conventional scalar PSA scheme. The setup was modified such that all of the three waves were co-polarized at the input of the waveguide and the polarizer was removed. For the same input power level of $21.3 \mathrm{dBm}$, only a $2 \mathrm{~dB}$ PSER was recorded.

To put these PSER figures into perspective, we simulated the phase transfer functions that correspond to the PSERs measured for the PA-PSA (PSER $=28.6 \mathrm{~dB}$ ) and the scalar PSA (PSER $=2 \mathrm{~dB}$ ) configurations, as shown in Fig. 5(top). It can be seen that the larger PSER value of the PA-PSA scheme allowed the transfer function to reach close to an ideal binary step function where for almost all values of input phase, the output phase is restricted to values close to either 0 or $\pi$. In contrast, the very limited PSER of the scalar PSA resulted in an almost linear transfer characteristic with rather insignificant phase quantization behavior. To further visualise the contrast between these two PSER values in the context of phase squeezing, we numerically applied these PSERs to a continuously phase-modulated signal and observed the resultant phase-squeezed output. Figure 5(bottom) illustrates the clear advantage of the higher PSER $(\sim 29 \mathrm{~dB})$ achieved through the PS-PSA, where the level of phase squeezing achieved is significantly superior to that obtained from the scalar PSA (2 dB).

These numerical predictions were confirmed experimentally by measuring constellation diagrams before and after the two PSA configurations. Constellation analysis was facilitated using homodyne coherent detection followed by real time data analysis (using a commercial OMA). Figure 6presents measured constellations for the continuously modulated input
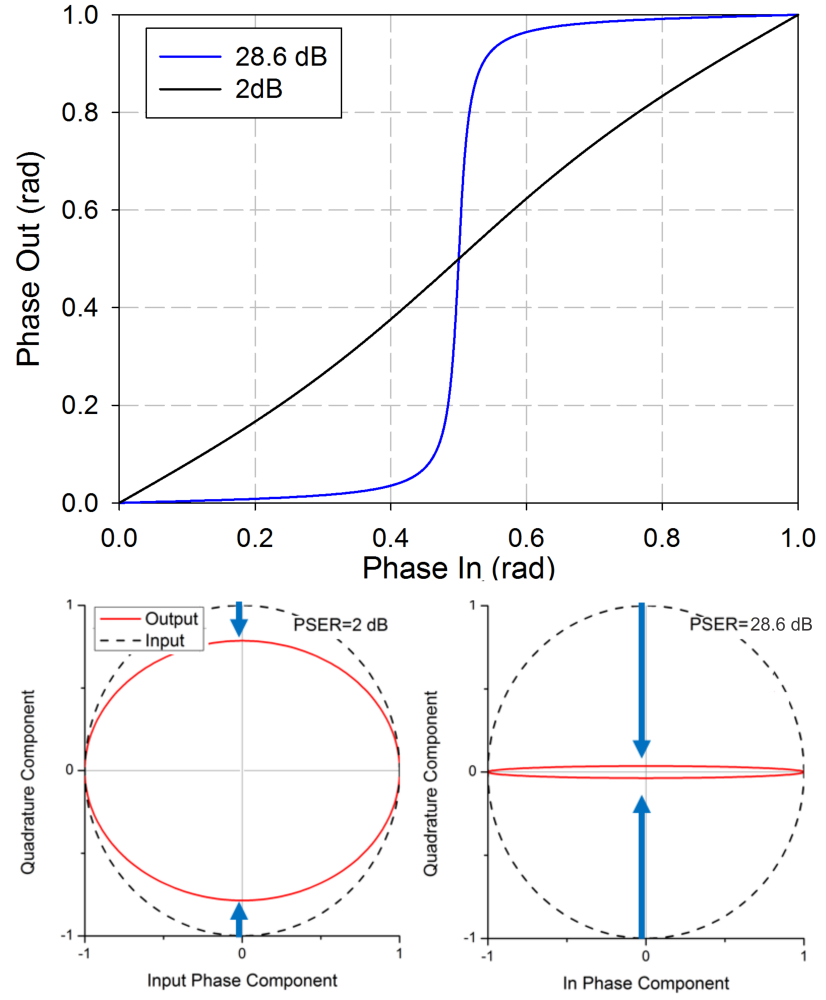

Fig. 5. Top: Numerically calculated phase transfer functions of the PA-PSA (blue) and the scalar PSA (black). Bottom: Constellation diagrams illustrating the difference in phase squeezing between the scalar PSA (bottom left)and PA-PSA (bottom right).

signal as well as those collected at the output, both for the PAPSA and scalar PSA schemes. The figure clearly highlights the excellent phase squeezing achievable through the large PSER in the case of the PA-PSA scheme. In contrast, the constellation at the output of the scalar PSA barely resembles an ellipse, illustrating the limited phase squeezing achievable at these power levels.

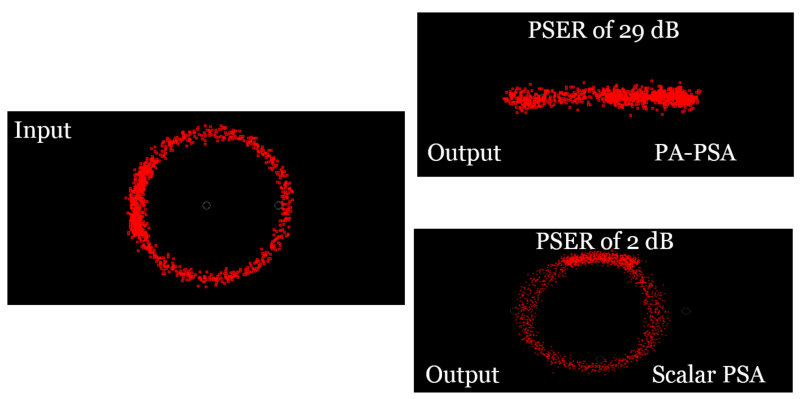

Fig. 6. Constellation diagrams at the input (left) and output of the PA-PSA (right top) and scalar PSA (right bottom).

\section{B. Phase Regeneration of $20 \mathrm{~Gb} / \mathrm{s}$ DPSK Signals}

The experimental setup of the PA-PSA-based phase quantizer of Fig. 3 was modified in order to study the phase regeneration of a data-modulated 20-Gb/s DPSK signal. The phase modulator in the signal path was replaced by a $20 \mathrm{~Gb} / \mathrm{s}$ DPSK transmitter driven by a $2^{15}-1$ pseudo-random bit sequence. 
The data signal was also sent through a noise module to emulate the effects of broadband phase noise. In this module, phase noise was generated by detecting amplified spontaneous emission (ASE) with a photo detector whose output was further amplified and fed to a phase modulator. Using the same phase modulator we also added a sinusoidal phase dither to the signal used in a feedback system to lock the phase of the pumps to the signal. Any slow thermo-acoustic relative phase drifts between the pumps and the signal were thus suppressed by controlling a piezo-electric transducer based fiber stretcher located in the path of Pump 2. In order to precisely adjust the polarization angle required for achieving maximum PSER and control real-time polarization fluctuations, we incorporated an additional feedback mechanism comprising a computer routine that monitored the noise statistics of the regenerated signal (supplied from the optical modulation analyser, OMA) and correspondingly adjusted the polarization angle of a computercontrolled polarization controller in order to optimize the projection angle to the polarizer, achieve a higher PSER and thus better noise (regeneration) performance. The data signal was detected using an optically pre-amplified homodyne coherent receiver followed by real time data analysis. Figure 7 shows a typical optical spectrum measured at the output of the waveguide.

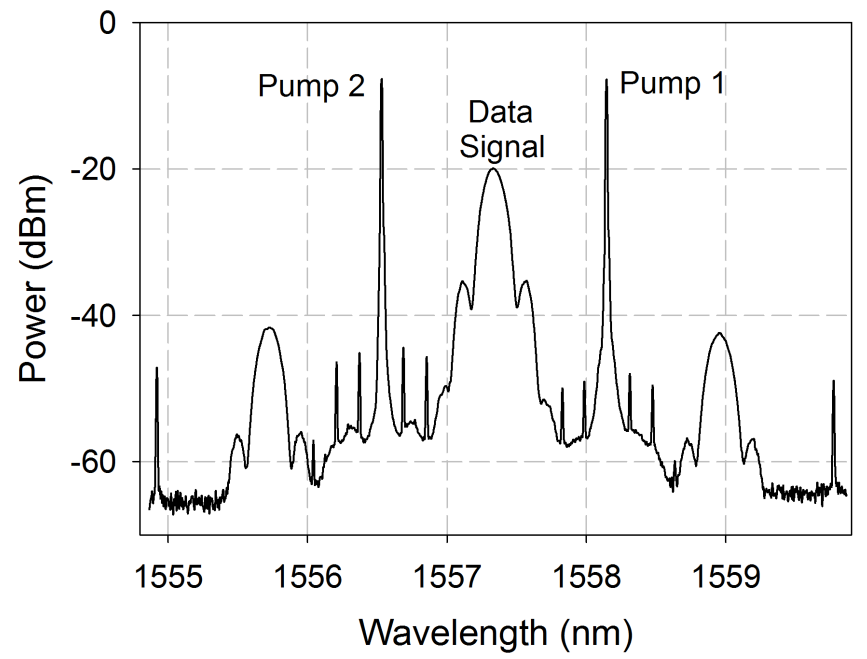

Fig. 7. Typical optical spectrum at the output of the waveguide.

Figure 8 shows the constellation diagrams collected before (top) and after (bottom) regeneration for two levels of phase noise. In the case of no added noise, the regenerated signal shows some degradation with respect to the back-to-back (B2B) case (which was assessed directly after the DPSK transmitter and before the EDFA). This slight deterioration could be attributed to two factors: Firstly, the introduction of ASE noise to the signal from the EDFA introduced just prior to coupling the signal to the waveguide. Secondly, the reduction of the OSNR of the received signal at the output of the polarizer due to the relatively low pump powers used, implying that the polarizer's transmission axis was very close to the polarization axis of the idler. Both factors contributed to the introduction of some magnitude error and a slight reduction in the overall quality of the generated signal. Both Cases A and
B of Fig. 8 however, in which phase noise of $11^{\circ} \mathrm{rms}$ and $26^{\circ}$ rms was introduced respectively to the signal, demonstrate that substantial phase squeezing and a significant improvement in the quality of the signal was achieved, albeit with some small magnitude noise degradation.
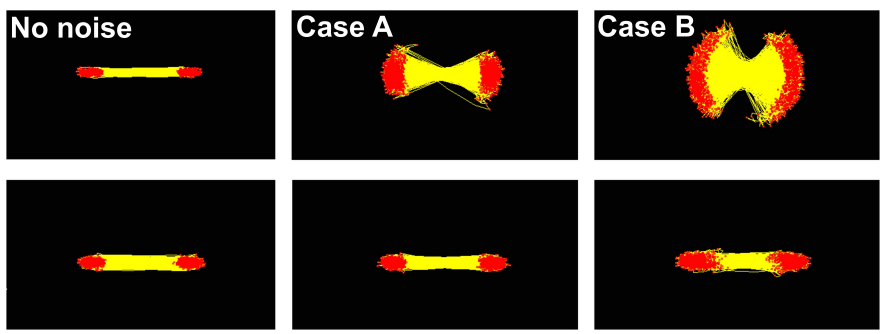

Fig. 8. Constellation diagrams before (top) and after (bottom) regeneration for three different phase noise levels.

The regenerative capability of the system was also confirmed through BER measurements, see Fig. 9 (note that the measurements were limited to a $\mathrm{BER}>10^{-5}$ due to OMAbased measuring system). As expected, the introduction of some amplitude noise results in a small $(0.3 \mathrm{~dB})$ BER penalty in the no-added-noise case. However, a $2 \mathrm{~dB}$ receiver sensitivity improvement was obtained in the case of the most severe phase noise (Case B) level (Fig. 9) with no observed errorfloor.

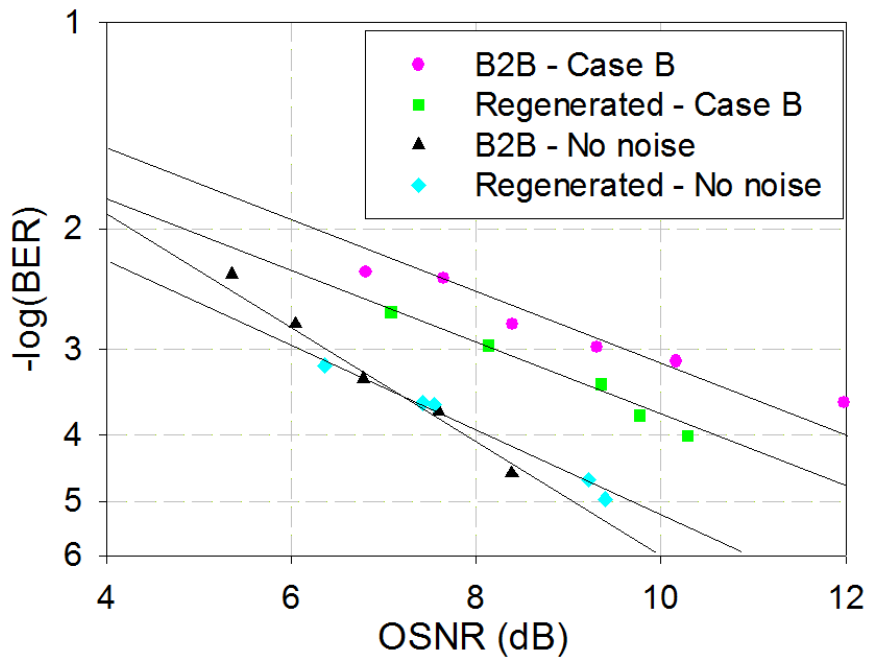

Fig. 9. BER measurements before and after regeneration for no extra noise induced and the most severe (Case B) noise case shown in Fig. 6 [23]

The improvements observed from the BER measurements were confirmed by comparing error vector magnitude (EVM), magnitude and phase-error statistics before and after regeneration, which allowed for a better understanding of the nature of the noise (Fig. 10. It can be seen from the figure that the no-added-noise case (the leftmost data point in the three graphs) shows a very small penalty due to the presence of the regenerator. All tests that included the addition of phase noise of a varying strength showed a universal improvement in the EVM and phase-error of the data signal.

The figure also reveals that the EVM shows a factor of almost 3 improvement and the level of phase-error is reduced 
by a factor of 6 in the case of the most severe noise level $\left(32.1^{\circ} \mathrm{rms}\right)$, confirming the capability of the regenerator to reduce phase noise and improve overall signal quality. Figure 10 (bottom) finally shows that a slight deterioration in the magnitude error of the regenerated signals as a function of increasing input phase noise error is observed (the input magnitude error remained constant at $\sim 10 \%$ in all cases), mainly due to the amplitude-noise-inducing factors outlined above as well as to the phase-to-amplitude noise conversion occurring in unsaturated PSAs [3].
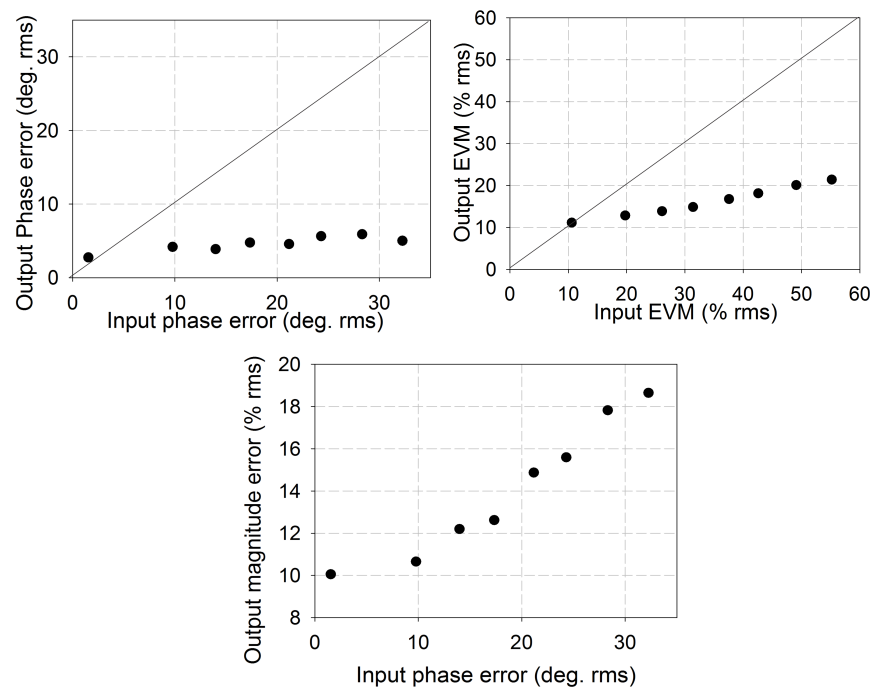

Fig. 10. Measured noise statistics of the signal before and after regeneration for different phase noise levels.

\section{Conclusion}

We have demonstrated a low-power CW-pumped PSA-based phase regenerator in a passive low-birefringence silicon-based waveguide. A SiGe device with an almost identical TE/TM behavior and a large nonlinear coefficient was designed and fabricated and used in a polarization-assisted phase sensitive amplifier scheme. A record PSER of approximately $29 \mathrm{~dB}$ was demonstrated in the $20 \mathrm{~mm}$-long waveguide using $21.3 \mathrm{dBm}$ of total power.

The waveguide performance was assessed in a phase regeneration experiment, revealing excellent phase squeezing qualities and achieving a six-fold reduction in phase noise and a BER improvement of approximately $2 \mathrm{~dB}$ in the regenerated signal for the most severe case of phase noise studied $\left(26^{\circ}\right.$ rms phase error). We envisage that a further enhancement in the performance of the regenerator could be obtained if an additional amplitude squeezing stage (following the phase regenerator) is added [24] or if the PA-PSA is operated in saturation. With respect to the latter route, we note that improving the coupling efficiency into the waveguide would allow higher power levels to be used, approaching the TPA threshold of the waveguide. Alternatively, adoption of a waveguide with a smaller cross-section and/or a graded Ge concentration profile [15] would further enhance the nonlinear phase shift and significantly boost the non-linear interactions between the waves.
The results reported here further demonstrate the versatility of SiGe devices and their emergence as a competitive alloptical signal processing platform. The addition of germanium to the waveguides adds an additional and valuable degree of freedom in the waveguide design, making $\mathrm{SiGe}$ devices a promising candidate for a wide range of all-optical signal processing applications.

\section{REFERENCES}

[1] D. J. Richardson, "Filling the light pipe," Science, vol. 330, no. 6002, pp. 327-328, 2010.

[2] Z. Tong, C. Lundström, P. Andrekson, C. McKinstrie, M. Karlsson, D. Blessing, E. Tipsuwannakul, B. Puttnam, H. Toda, and L. GrünerNielsen, "Towards ultrasensitive optical links enabled by low-noise phase-sensitive amplifiers," Nature Photonics, vol. 5, no. 7, pp. 430436, 2011.

[3] R. Slavík, F. Parmigiani, J. Kakande, C. Lundström, M. Sjödin, P. A. Andrekson, R. Weerasuriya, S. Sygletos, A. D. Ellis, L. Grüner-Nielsen et al., "All-optical phase and amplitude regenerator for next-generation telecommunications systems," Nature Photonics, vol. 4, no. 10, pp. 690695, 2010.

[4] J. Kakande, R. Slavík, F. Parmigiani, A. Bogris, D. Syvridis, L. GrünerNielsen, R. Phelan, P. Petropoulos, and D. J. Richardson, "Multilevel quantization of optical phase in a novel coherent parametric mixer architecture," Nature Photonics, vol. 5, no. 12, pp. 748-752, 2011.

[5] R. Slavík, A. Bogris, F. Parmigiani, J. Kakande, M. Westlund, M. Skold, L. Gruner-Nielsen, R. Phelan, D. Syvridis, P. Petropoulos et al., "Coherent all-optical phase and amplitude regenerator of binary phase-encoded signals," Selected Topics in Quantum Electronics, IEEE Journal of, vol. 18 , no. 2, pp. 859-869, 2012.

[6] P. Andrekson, "Progress in phase-sensitive fiber-optic parametric amplifiers and their applications," in CLEO: Science and Innovations. Optical Society of America, 2011, p. CWD1.

[7] J. Leuthold, C. Koos, and W. Freude, "Nonlinear silicon photonics," Nature Photonics, vol. 4, no. 8, pp. 535-544, 2010.

[8] B. G. Lee, A. Biberman, A. C. Turner-Foster, M. Foster, M. Lipson, A. L. Gaeta, K. Bergman et al., "Demonstration of broadband wavelength conversion at $40 \mathrm{~Gb} / \mathrm{s}$ in silicon waveguides," Photonics Technology Letters, IEEE, vol. 21, no. 3, pp. 182-184, 2009.

[9] E. Dulkeith, Y. A. Vlasov, X. Chen, N. C. Panoiu, R. M. Osgood Jr et al., "Self-phase-modulation in submicron silicon-on-insulator photonic wires," Optics Express, vol. 14, no. 12, pp. 5524-5534, 2006.

[10] H. Rong, A. Liu, R. Jones, O. Cohen, D. Hak, R. Nicolaescu, A. Fang, and M. Paniccia, "An all-silicon Raman laser," Nature, vol. 433, no. 7023, pp. 292-294, 2005.

[11] R. K. Lau, M. R. Lamont, A. G. Griffith, Y. Okawachi, M. Lipson, and A. L. Gaeta, "Octave-spanning mid-infrared supercontinuum generation in silicon nanowaveguides," Optics Letters, vol. 39, no. 15, pp. 45184521,2014

[12] X. Liu, B. Kuyken, G. Roelkens, R. Baets, R. M. Osgood Jr, and W. M. Green, "Bridging the mid-infrared-to-telecom gap with silicon nanophotonic spectral translation," Nature Photonics, vol. 6, no. 10, pp. 667-671, 2012.

[13] K. Hammani, M. A. Ettabib, A. Bogris, A. Kapsalis, D. Syvridis, M. Brun, P. Labeye, S. Nicoletti, D. J. Richardson, and P. Petropoulos, "Optical properties of silicon germanium waveguides at telecommunication wavelengths," Optics Express, vol. 21, no. 14, pp. 16690-16701, 2013.

[14] M. A. Ettabib, K. Hammani, F. Parmigiani, L. Jones, A. Kapsalis, A. Bogris, D. Syvridis, M. Brun, P. Labeye, S. Nicoletti et al., "Fwmbased wavelength conversion of 40 Gbaud PSK signals in a silicon germanium waveguide," Optics Express, vol. 21, no. 14, pp. 16683$16689,2013$.

[15] K. Hammani, M. A. Ettabib, A. Bogris, A. Kapsalis, D. Syvridis, M. Brun, P. Labeye, S. Nicoletti, and P. Petropoulos, "Towards nonlinear conversion from mid-to near-infrared wavelengths using silicon germanium waveguides," Optics Express, vol. 22, no. 8, pp. 9667-9674, 2014.

[16] M. A. Ettabib, L. Xu, A. Bogris, A. Kapsalis, M. Belal, E. Lorent, P. Labeye, S. Nicoletti, K. Hammani, D. Syvridis et al., "Broadband telecom to mid-infrared supercontinuum generation in a dispersionengineered silicon germanium waveguide," Optics Letters, vol. 40, no. 17 , pp. 4118-4121, 2015. 
[17] Y. Zhang, C. Husko, J. Schröder, S. Lefrancois, I. H. Rey, T. F. Krauss, and B. J. Eggleton, "Phase-sensitive amplification in silicon photonic crystal waveguides," Optics Letters, vol. 39, no. 2, pp. 363-366, 2014.

[18] F. Da Ros, D. Vukovic, A. Gajda, K. Dalgaard, L. Zimmermann, B. Tillack, M. Galili, K. Petermann, and C. Peucheret, "Phase regeneration of DPSK signals in a silicon waveguide with reverse-biased pin junction," Optics Express, vol. 22, no. 5, pp. 5029-5036, 2014.

[19] M. A. Ettabib, A. Kapsalis, A. Bogris, F. Parmigiani, V. J. F. Rancao, K. Bottrill, M. Brun, P. Labeye, S. Nicoletti, K. Hammani, D. Syvridis, D. J. Richardson, and P. Petropoulos, "Polarization insensitive wavelength conversion in a low-birefringence sige waveguide," IEEE Photonics Technology Letters, vol. 28, no. 11, pp. 1221-1224, 2016.

[20] F. Parmigiani, G. D. Hesketh, R. Slavik, P. Horak, P. Petropoulos, and D. J. Richardson, "Optical phase quantizer based on phase sensitive four wave mixing at low nonlinear phase shifts," Photonics Technology Letters, IEEE, vol. 26, no. 21, pp. 2146-2149, 2014.

[21] F. Parmigiani, G. Hesketh, R. Slavík, P. Horak, P. Petropoulos, and D. J. Richardson, "Polarization-assisted phase-sensitive processor," Journal of Lightwave Technology, vol. 33, no. 6, pp. 1166-1174, 2015.

[22] M. A. Ettabib, F. Parmigiani, A. Kapsalis, A. Bogris, M. Brun, P. Labeye, S. Nicoletti, K. Hammani, D. Syvridis, P. Petropoulos et al., "Record phase sensitive extinction ratio in a silicon germanium waveguide," in CLEO: Science and Innovations. Optical Society of America, 2015, pp. STh1O-8.

[23] M. Ettabib, K. Bottrill, F. Parmigiani, A. Kapsalis, A. Bogris, M. Brun, P. Labeye, S. Nicoletti, K. Hammani, D. Syvridis, D. J. Richardson, and P. Petropoulos, "PSA-based phase regeneration of DPSK signals in a silicon germanium waveguide," in Optical Communication (ECOC), 2015 European Conference on, 2015, pp. 1-3.

[24] K. R. H. Bottrill, F. Parmigiani, L. Jones, G. Hesketh, D. J. Richardson, and P. Petropoulos, "Phase and amplitude regeneration through sequential PSA and FWM saturation in HNLF," in Optical Communication (ECOC), 2015 European Conference on, Sept 2015, pp. 1-3. 